


\title{
ON THE COEFFICIENTS OF DIFFERENTIATED EXPANSIONS OF ULTRASPHERICAL POLYNOMIALS
}

\author{
Andreas Karageorghis ${ }^{1}$ \\ Mathematics Department \\ Southern Methodist University \\ Dallas, TX 75275-0156 \\ and \\ Timothy N. Phillips ${ }^{2}$ \\ Department of Mathematics \\ University College of Wales \\ Aberystwyth, SY23 3BZ, U.K.
}

\begin{abstract}
A formula expressing the coefficients of an expansion of ultraspherical polynomials which has been differentiated an arbitrary number of times in terms of the coefficients of the original expansion is proved. The particular examples of Chebyshev and Legendre polynomials are considered.
\end{abstract}

\footnotetext{
${ }^{1}$ Part of this work was performed while this author was an SERC research fellow at the University College of Wales, Aberystwyth, U.K.

${ }^{2}$ Research was supported in part for the second author by the National Aeronautics and Space Administration under NASA Contract No. NAS1-18605 while he was in residence at the Institute for Computer Applications in Science and Engineering (ICASE), NASA Langley Research Center, Hampton, VA 23665.
} 



\section{Introduction}

The current popularity and interest in spectral methods is mainly due to their superior approximation properties compared with other methods of discretization. One can show that if the eigenfunctions of a singular Sturm-Liouville problem are used as basis functions then the rate of decay of the expansion coefficients is determined by the smoothness of the function being expanded and not by any special boundary conditions satisfied by the function. If the functions of interest are infinitely differentiable then the nth coefficient $a_{n}$ decreases faster than any finite power of $\frac{1}{n}$ as $n \rightarrow \infty[2]$.

Spectral methods have been extensively used in the solution of boundary value problems and in computational fluid dynamics $[1,2]$. In most of these applications use is made of formulae relating the expansion coefficients of derivatives appearing in the differential equation to those of the function itself. In fact, the coefficients of successive derivatives of a function are related by a recurrence relation which can be solved numerically in an efficient and stable way. This is a distinguishing feature of the pseudospectral method [1]. For the Galerkin and tau variants of the spectral method explicit expressions for the expansion coefficients of derivatives in terms of the expansion coefficients of the solution are required. In this paper, we obtain such expressions when the basis functions are ultraspherical polynomials. This work generalizes earlier results for the Chebyshev and Legendre polynomials $[3,4]$ and is the converse of a result on the coefficients of integrated expansions of ultraspherical polynomials [5].

In Section 2 we give relevant properties of ultraspherical polynomials and in Section 3 we describe how they are used to solve boundary value problems with the tau method. In Section 4 we prove the main result of the paper which is an expression for the coefficients of a general order derivative of an expansion in ultraspherical polynomials in terms of the coefficients of the original expansion. Concluding remarks are given in Section 5.

\section{Properties of Ultraspherical Polynomials}

The ultraspherical or Gegenbauer (see [6]) polynomials $\left\{P_{n}^{(\gamma)}(x)\right\}_{n=0}^{\infty}$ are orthogonal on the interval $[-1,1]$ with respect to the weight function $w_{\gamma}(x)=\left(1-x^{2}\right)^{\gamma}$, i.e.,

$$
\int_{-1}^{1}\left(1-x^{2}\right)^{\gamma} P_{m}^{(\gamma)}(x) P_{n}^{(\gamma)}(x) d x=0, \quad m \neq n .
$$

In order that the weight function is non-negative and integrable we assume $\gamma>-1$. The ultraspherical polynomials are eigenfunctions of the following singular Sturm-Liouville problem

$$
\left(1-x^{2}\right) \phi^{\prime \prime}(x)-2(\gamma+1) x \phi^{\prime}(x)+n(n+2 \gamma+1) \phi(x)=0 .
$$

A consequence of this is that spectral accuracy can be achieved for expansions in ultraspherical polynomials. This class of orthogonal polynomials is a subclass of the Jacobi polynomials.

Let $f(x)$ be an infinitely differentiable function defined on $[-1,1]$. We may represent $f(x)$ in the form

$$
f(x)=\sum_{n=0}^{\infty} a_{n} P_{n}^{(\gamma)}(x)
$$


where $P_{n}^{(\gamma)}(x)$ is the ultraspherical polynomial of degree $n$. Further, let $a_{n}^{(q)}$ denote the ultraspherical coefficients of the qth derivative of $f(x)$ then it can be shown [5] that the coefficients of successive derivatives of $f(x)$ satisfy the recurrence relation

$$
a_{n}^{(q-1)}=\frac{(n+2 \gamma)}{(n+\gamma)(2 n+2 \gamma-1)} a_{n-1}^{(q)}-\frac{(n+\gamma+1)}{(n+2 \gamma+1)(2 n+2 \gamma+3)} a_{n+1}^{(q)} .
$$

This recurrence relation may be solved to yield

$$
a_{n}^{(q)}=(2 n+2 \gamma+1) \frac{\Gamma(n+2 \gamma+1)}{\Gamma(n+\gamma+1)} \sum_{k=1}^{\infty} \frac{\Gamma(n+\gamma+2 k)}{\Gamma(n+2 \gamma+2 k)} a_{n+2 k-1}^{(q-1)},
$$

for $q \geq 1$ where $a_{n}^{(0)}=a_{n}$ and $\Gamma(\cdot)$ is the Gamma function.

\section{The Tau Method for a Model Problem}

Consider the boundary value problem

$$
\begin{gathered}
u^{\prime \prime}(x)+\lambda u(x)=g(x), \quad x \in[-1,1], \\
u( \pm 1)=0,
\end{gathered}
$$

where $\lambda$ is a real scalar. Suppose that we approximate $u(x)$ by a truncated expansion of ultraspherical polynomials

$$
u_{N}(x)=\sum_{n=0}^{N} a_{n} P_{n}^{(\gamma)}(x)
$$

We seek to determine $a_{n}(0 \leq n \leq N)$ using the tau method.

The coefficients $a_{n}$ are chosen so that $u_{N}(x)$ satisfies

$$
u_{N}^{\prime \prime}(x)+\lambda u_{N}(x)=g_{N}(x)
$$

where

$$
g_{N}(x)=\sum_{n=0}^{N} d_{n} P_{n}^{(\gamma)}(x)
$$

Substituting (8) and (10) into (9), multiplying by $w_{\gamma}(x) P_{m}^{(\gamma)}(x), \quad 0 \leq m \leq N-2$, and integrating over $[-1,1]$ yields

$$
a_{m}^{(2)}+\lambda a_{m}=d_{m}, \quad 0 \leq m \leq N-2 .
$$

Two further equations are obtained from the boundary conditions by inserting (8) into (7):

$$
\sum_{n=0}^{N} a_{n}( \pm 1)^{n} \frac{\Gamma(n+\gamma+1)}{\Gamma(\gamma+1)}=0
$$

Thus (11) and (12) comprise $N+1$ equations for the $N+1$ unknown coefficients $a_{n}(0 \leq n \leq$ $N)$. 
The coefficients, $a_{n}^{(2)}$, of the second derivative of the approximation $u_{N}(x)$ are related to the coefficients, $a_{n}$, of $u_{N}(x)$ by invoking (4) with $q=1$ and $q=2$. In the next section we show how the coefficients of any derivative may be expressed in terms of the original expansion coefficients. This allows us, for example, to replace $a_{m}^{(2)}$ in (11) by an explicit expression in terms of the $a_{n}$. In this way we can set up a linear system for $a_{n}(0 \leq n \leq N)$ which may be solved using standard direct solvers.

\section{The Theorem and its Proof}

\section{Theorem}

Let $f(x)$ be an infinitely differentiable function defined on the interval [-1,1]. The coefficients $a_{n}^{(q)}$ of an expansion of ultraspherical polynomials of the qth derivative of $f(x)$ are related to the coefficients $a_{n}$ of an expansion of ultraspherical polynomials of $f(x)$ by

$$
\begin{aligned}
a_{n}^{(q)}= & \frac{(2 n+2 \gamma+1) \Gamma(n+2 \gamma+1)}{2^{q-2}(q-1) ! \Gamma(n+\gamma+1)} \sum_{k=1}^{\infty} \frac{\Gamma(n+\gamma+2 k+q-1)}{\Gamma(n+2 \gamma+2 k+q-1)} \frac{(k+q-2) !}{(k-1) !} \\
& \times \frac{\Gamma(2 n+2 \gamma+2 k+2 q-2)}{\Gamma(2 n+2 \gamma+2 k+1)} \quad \frac{\Gamma(n+\gamma+k+1)}{\Gamma(n+\gamma+k+q-1)} a_{n+2 k+q-2},
\end{aligned}
$$

for all $n \geq 0, q \geq 1$.

\section{Proof}

For $q=1$ (13) reduces to (5) when $q=1$. We proceed by induction and assume that the theorem is valid for $q$. We need to show that it is valid for $q+1$, i.e.,

$$
\begin{aligned}
a_{n}^{(q+1)}= & \frac{(2 n+2 \gamma+1) \Gamma(n+2 \gamma+1)}{2^{q-1} q ! \Gamma(n+\gamma+1)} \sum_{k=1}^{\infty} \frac{\Gamma(n+\gamma+2 k+q)(k+q-1) ! \Gamma}{\Gamma(n+2 \gamma+2 k+q)} \\
& \frac{(2 n+2 \gamma+2 k+2 q) \Gamma(n+\gamma+k+1)}{(k-1) ! \Gamma(2 n+2 \gamma+2 k+1) \Gamma(n+\gamma+k+q)} a_{n+2 k+q-1} .
\end{aligned}
$$

We replace $q$ by $q+1$ in (5) and then substitute for $a_{n}^{(q)}$ using (13), which is the inductive hypothesis, to give

$$
\begin{gathered}
a_{n}^{(q+1)}=(2 n+2 \gamma+1) \frac{\Gamma(n+2 \gamma+1)}{\Gamma(n+\gamma+1)} \sum_{k=1}^{\infty} \frac{\Gamma(n+\gamma+2 k)}{\Gamma(n+2 \gamma+2 k)}\left[\frac{(2 n+2 \gamma+4 k-1)}{2^{q-2}(q-1) !} \frac{\Gamma(n+2 \gamma+2 k)}{\Gamma(n+\gamma+k)}\right. \\
\times \sum_{j=1}^{\infty} \frac{\Gamma(n+\gamma+2 k+2 j+q-2)(j+q-2) !}{\Gamma(n+2 \gamma+2 k+2 j+q-2)(j-1) !} \\
\frac{\Gamma(2 n+2 \gamma+4 k+2 j+2 q-4) \Gamma(n+\gamma+2 k+j)}{\Gamma(2 n+2 \gamma+4 k+2 j-1) \Gamma(n+\gamma+2 k+j+q-2)} a_{n+2 k+2 j+q-3} \\
=\frac{(2 n+2 \gamma+1) \Gamma(n+2 \gamma+1)}{2^{q-2}(q-1) ! \Gamma(n+\gamma+1)} \sum_{k=1}^{\infty}(2 n+2 \gamma+4 k-1) \sum_{j=1}^{\infty} \frac{\Gamma(n+\gamma+2 k+2 j+q-2)}{\Gamma(n+2 \gamma+2 k+2 j+q-2)}
\end{gathered}
$$




$$
\frac{(j+q-2) ! \Gamma(2 n+2 \gamma+4 k+2 j+2 q-4)}{(j-1) ! \Gamma(2 n+\gamma+4 k+2 j-1)} \times \frac{\Gamma(n+\gamma+2 k+j)}{\Gamma(n+\gamma+2 k+j+q-2)} a_{n+2 k+2 j+q-3} .
$$

If we write $k+j=\ell+1$ then we may write $a_{n}^{(q+1)}$ in the form

$$
\begin{aligned}
a_{n}^{(q+1)}= & \frac{(2 n+2 \gamma+1) \Gamma(n+2 \gamma+1)}{2^{q-2}(q-1) ! \Gamma(n+\gamma+1)} \sum_{\ell=1}^{\infty} \frac{\Gamma(n+\gamma+2 \ell+q)}{\Gamma(n+2 \gamma+2 \ell+q)}\left[\sum_{k=1}^{\ell}(2 n+2 \gamma+4 k-1)\right. \\
& \left.\times \frac{(\ell-k+q-1) !}{(\ell-k) !} \quad \frac{\Gamma(2 n+2 \gamma+2 k+2 \ell+2 q-2) \Gamma(n+\gamma+k+\ell+1)}{\Gamma(2 n+2 \gamma+2 k+2 \ell+1) \Gamma(n+\gamma+k+\ell+q-1)}\right] a_{n+2 \ell+q-1} .
\end{aligned}
$$

In order to complete the proof of the theorem we need to prove the following lemma:

Lemma

$$
\begin{aligned}
\sum_{k=1}^{\ell}(2 n+2 \gamma+ & 4 k-1) \frac{(\ell-k+q-1) ! \Gamma(2 n+2 \gamma+2 k+2 \ell+2 q-2) \Gamma(n+\gamma+k+\ell+1)}{(\ell-k) ! \Gamma(2 n+2 \gamma+2 k+2 \ell+1) \Gamma(n+\gamma+k+\ell+q-1)} \\
= & \frac{1}{2 q} \frac{(\ell+q-1) ! \Gamma(2 n+2 \gamma+2 \ell+2 q) \Gamma(n+\gamma+\ell+1)}{(\ell-1) ! \Gamma(2 n+2 \gamma+2 \ell+1) \Gamma(n+\gamma+\ell+q)},(16)
\end{aligned}
$$

for all values of $n \geq 0, \ell \geq 1, q \geq 1$.

$\underline{\text { Proof }}$

When $\ell=1$ both sides of (16) are equal, taking the value

$$
\frac{(q-1) ! \Gamma(2 n+2 \gamma+2 q+2) \Gamma(n+\gamma+2)}{2 \Gamma(n+\gamma+q+1) \Gamma(2 n+2 \gamma+3)},
$$

and in this case (16) holds for all values of $n \geq 0, q \geq 1$. The proof of the lemma proceeds by induction on $\ell$. We need to prove that (16) is true when $\ell$ is replaced by $\ell+1$, i.e.,

$$
\begin{aligned}
\sum_{k=1}^{\ell+1}(2 n+2 \gamma & +4 k-1) \frac{(\ell-k+q) ! \Gamma(2 n+2 \gamma+2 k+2 \ell+2 q) \Gamma(n+\gamma+k+\ell+2)}{(\ell-k+1) ! \Gamma(2 n+2 \gamma+2 k+2 \ell+3) \Gamma(n+\gamma+k+\ell+q)} \\
& =\frac{1}{2 q} \frac{(\ell+q) !}{\ell !} \frac{\Gamma(2 n+2 \gamma+2 q+2) \Gamma(n+\gamma+\ell+2)}{\Gamma(2 n+2 \gamma+2 \ell+3) \Gamma(n+\gamma+\ell+q+1)} .
\end{aligned}
$$

If we replace $n$ by $n+2$ in (17) we obtain

$$
\begin{aligned}
\sum_{k=1}^{\ell}(2 n+2 \gamma+ & 4 k+3) \frac{(\ell-k+q-1) !}{(\ell-k) !} \frac{\Gamma(2 n+2 \gamma+2 k+2 \ell+2 q+2) \Gamma(n+\gamma+k+\ell+3)}{\Gamma(2 n+2 \gamma+2 k+2 \ell+5) \Gamma(n+\gamma+k+\ell+q+2)} \\
& =\frac{1}{2 q} \frac{(\ell+q-1) !}{(\ell-1) !} \frac{\Gamma(2 n+2 \gamma+2 \ell+2 q+4) \Gamma(n+\gamma+\ell+3)}{\Gamma(2 n+2 \gamma+2 \ell+5) \Gamma(n+\gamma+\ell+q+2)}
\end{aligned}
$$


If we set $p=k+1$ then (18) becomes

$$
\begin{gathered}
\sum_{p=2}^{\ell+1}(2 n+2 \gamma+4 p-1) \frac{(\ell-p+q) ! \Gamma(2 n+2 \gamma+2 p+2 \ell+2 q) \Gamma(n+\gamma+p+\ell+2)}{(\ell-p+1) ! \Gamma(2 n+2 \gamma+2 p+2 \ell+3) \Gamma(n+\gamma+p+\ell+q)} \\
=\frac{1}{2 q} \frac{(\ell+q-1) ! \Gamma(2 n+2 \gamma+2 \ell+2 q+4) \Gamma(n+\gamma+\ell+3)}{(\ell-1) ! \Gamma(2 n+2 \gamma+2 \ell+5) \Gamma(n+\gamma+\ell+q+2)}
\end{gathered}
$$

Now the left-hand side of (17) may be written as

$$
\begin{gathered}
(2 n+2 \gamma+3) \frac{(\ell+q-1) ! \Gamma(2 n+2 \gamma+2 \ell+2 q+2) \Gamma(n+\gamma+\ell+3)}{\ell ! \Gamma(2 n+2 \gamma+2 \ell+5) \Gamma(n+\gamma+\ell+q+1)} \\
+\sum_{k=2}^{\ell+1}(2 n+2 \gamma+4 k-1) \frac{(\ell-k+q) ! \Gamma(2 n+2 \gamma+2 k+2 \ell+2 q) \Gamma(n+\gamma+k+\ell+2)}{(\ell-k+1) ! \Gamma(2 n+2 \gamma+2 k+2 \ell+3) \Gamma(n+\gamma+k+\ell+q)}
\end{gathered}
$$

which, in view of (19), is equal to the following

$$
\begin{gathered}
(2 n+2 \gamma+3) \frac{(\ell+q-1) ! \Gamma(2 n+2 \gamma+2 \ell+2 q+2) \Gamma(n+\gamma+\ell+3)}{\ell ! \Gamma(2 n+2 \gamma+2 \ell+5) \Gamma(n+\gamma+\ell+q+1)} \\
+\frac{1}{2 q} \frac{(\ell+q-1) ! \Gamma(2 n+2 \gamma+2 \ell+2 q+4) \Gamma(n+\gamma+\ell+3)}{(\ell-1) ! \Gamma(2 n+2 \gamma+2 \ell+5) \Gamma(n+\gamma+\ell+q+2)} \\
=\frac{1}{2 q} \frac{(\ell+q-1) ! \Gamma(2 n+2 \gamma+2 \ell+2 q+2) \Gamma(n+\gamma+\ell+3)}{(\ell-1) ! \Gamma(2 n+2 \gamma+2 \ell+5) \Gamma(n+\gamma+\ell+q+1)} \\
\times\left[\frac{(2 n+2 \gamma+3) 2 q}{\ell}+\frac{(2 n+2 \gamma+2 \ell+2 q+3)(2 n+2 \gamma+2 \ell+2 q+2)}{(n+\gamma+\ell+q+1)}\right] \\
=\frac{1}{2 q} \frac{(\ell+q-1) ! \Gamma(2 n+2 \gamma+2 \ell+2 q+2) \Gamma(n+\gamma+\ell+3)}{(\ell-1) ! \Gamma(2 n+2 \gamma+2 \ell+5) \Gamma(n+\gamma+\ell+q+1)}\left[\frac{2(2 n+2 \gamma+2 \ell+3)(q+\ell)}{\ell}\right] \\
=\frac{1}{2 q} \frac{(\ell+q) !}{\ell !} \frac{\Gamma(2 n+2 \gamma+2 \ell+2 q+2) \Gamma(n+\gamma+\ell+2)}{\Gamma(2 n+2 \gamma+2 \ell+3) \Gamma(n+\gamma+\ell+q+1)},
\end{gathered}
$$

which is the right-hand side of (17). This completes the inductive step and proves the lemma.

Application of the lemma to the expression in square brackets on the right-hand side of (15) gives (14) which completes the proof of the theorem.

The particular expressions for the Chebyshev and Legendre polynomials may be derived as special cases of the theorem. We give these as corollaries to the main theorem.

Corollary 1 ([4])

If $f(x)$ is an infinitely differentiate function on $[-1,1]$ then the Legendre coefficients, $a_{n}^{(a)}$, of the qth derivative of $f(x)$ are related to the Legendre coefficients, $a_{n}$, of $f(x)$ by

$$
a_{n}^{(q)}=\frac{(2 n+1)}{2^{q-2}(q-1) !} \sum_{k=1}^{\infty} \frac{(k+q-2) !}{(k-1) !} \frac{(2 n+2 k+2 q-3) !(n+k) !}{(2 n+2 k) !(n+k+q-2) !} a_{n+2 k+q-2},
$$


for all $n \geq 0, q \geq 1$.

\section{$\underline{\text { Proof }}$}

Simply set $\gamma=0$ in (13).

Corollary 2 ([3])

The corresponding Chebyshev derivative coefficients are related by

$$
c_{n} b_{n}^{(q)}=\frac{2^{q}}{(q-1) !} \sum_{k=1}^{\infty} \frac{(k+q-2) !(n+k+q-2) !}{(k-1) !(n+k-1) !}(n+2 k+q-2) b_{n+2 k+q-2},
$$

where $c_{0}=2, c_{n}=1(n>0)$.

\section{Proof}

We consider the case $n>0$. The ultraspherical polynomials with $\gamma=-\frac{1}{2}$ are related to the Chebyshev polynomials by

$$
P_{n}^{(-1 / 2)}(x)=2^{-2 n}\left(\begin{array}{c}
2 n \\
n
\end{array}\right) T_{n}(x), \quad n \geq 1 .
$$

The ultraspherical coefficients, $a_{n}^{(q)}$, of the qth derivative of $f(x)$ when $\gamma=-\frac{1}{2}$ are therefore related to the $b_{n}^{(q)}$ by

$$
a_{n}^{(q)}=2^{2 n} \frac{(n !)^{2}}{(2 n) !} b_{n}^{(q)}, \quad \text { for all } q>0 .
$$

Setting $\gamma=-\frac{1}{2}$ and substituting $a_{n}^{(q)}$ from (13) into (22) we obtain

$$
\begin{gathered}
2^{2 n} \frac{(n !)^{2}}{(2 n) !} b_{n}^{(q)}=\frac{2 n \Gamma(n)}{2^{q-2}(q-1) !} \frac{1}{\Gamma\left(n+\frac{1}{2}\right)} \sum_{k=1}^{\infty} \frac{(k+q-2) ! \Gamma\left(n+2 k+q-\frac{3}{2}\right)}{(k-1) ! \Gamma(n+2 k+q-2)} \\
\times \frac{\Gamma(2 n+2 k+2 q-3) \Gamma\left(n+k+\frac{1}{2}\right)}{\Gamma(2 n+2 k) \Gamma\left(n+k+q-\frac{3}{2}\right)} \cdot 2^{2 n+4 k+2 q-4} \frac{[(n+2 k+q-2) !]^{2}}{(2 n+4 k+2 q-4) !} b_{n+2 k+q-2} .
\end{gathered}
$$

After a little manipulation we may write this in the form

$$
\begin{aligned}
b_{n}^{(q)}= & \frac{2^{q-1}}{(q-1) !}\left[\frac{(2 n) !}{n ! \Gamma\left(n+\frac{1}{2}\right)}\right] \sum_{k=1}^{\infty} \frac{(k+q-2) !}{(k-1) !}\left[\frac{\Gamma\left(n+2 k+q-\frac{3}{2}\right)(n+2 k+q-2) !}{(2 n+4 k+2 q-4) !}\right] \\
& \times\left[\frac{\Gamma\left(n+k+\frac{1}{2}\right)}{\Gamma(2 n+2 k)}\right]\left[\frac{(2 n+2 k+2 q-4) !}{\Gamma\left(n+k+q-\frac{3}{2}\right)}\right](n+2 k+q-2) 2^{4 k} b_{n+2 k+q-2} .
\end{aligned}
$$

Now application of the formula

$$
\frac{(2 m) !}{\Gamma\left(m+\frac{1}{2}\right)}=\frac{2^{2 m} m !}{\Gamma\left(\frac{1}{2}\right)}
$$


to each of the terms in square brackets on the right-hand side of (23) yields (21) for $n>0$. The special case $n=0$ may be derived in a similar way.

\section{Conclusions}

This paper proves formulae associated with the differentiation of expansions of ultraspherical polynomials and describes how they can be used to solve two-point boundary value problems. Formulae relating the coefficients in expansions which have been differentiated an arbitrary number of times to those in the original expansion are proved. 


\section{References}

[1] C. Canuto, M. Y. Hussaini, A. Quarteroni, and T. A. Zang, Spectral Methods in Fluid Dynamics, Springer-Verlag, Berlin, 1988.

[2] D. Gottlieb and S. A. Orszag, Numerical Analysis of Spectral Methods: Theory and Applications, CBMS-NSF Regional Conference Series in Applied Mathematics, 26, SIAM, Philadelphia, 1977.

[3] A. Karageorghis, "A note on the Chebyshev coefficients of the general order derivative of an infinitely differentiable function," J. Comput. Appl. Math., Vol. 21, 1988, pp. 129-132.

[4] T. N. Phillips, "On the Legendre coefficients of a general order derivative of an infinitely differentiable function," IMA J. Numer. Anal., Vol. 8, 1988, pp. 455-459.

[5] T. N. Phillips and A. Karageorghis, "On the coefficients of integrated expansions of ultraspherical polynomials," SIAM J. Numer. Anal., to appear.

[6] A. Erdelyi (Ed.), Higher Transcendental Functions Vol. II, McGraw-Hill, New York, 1953. 


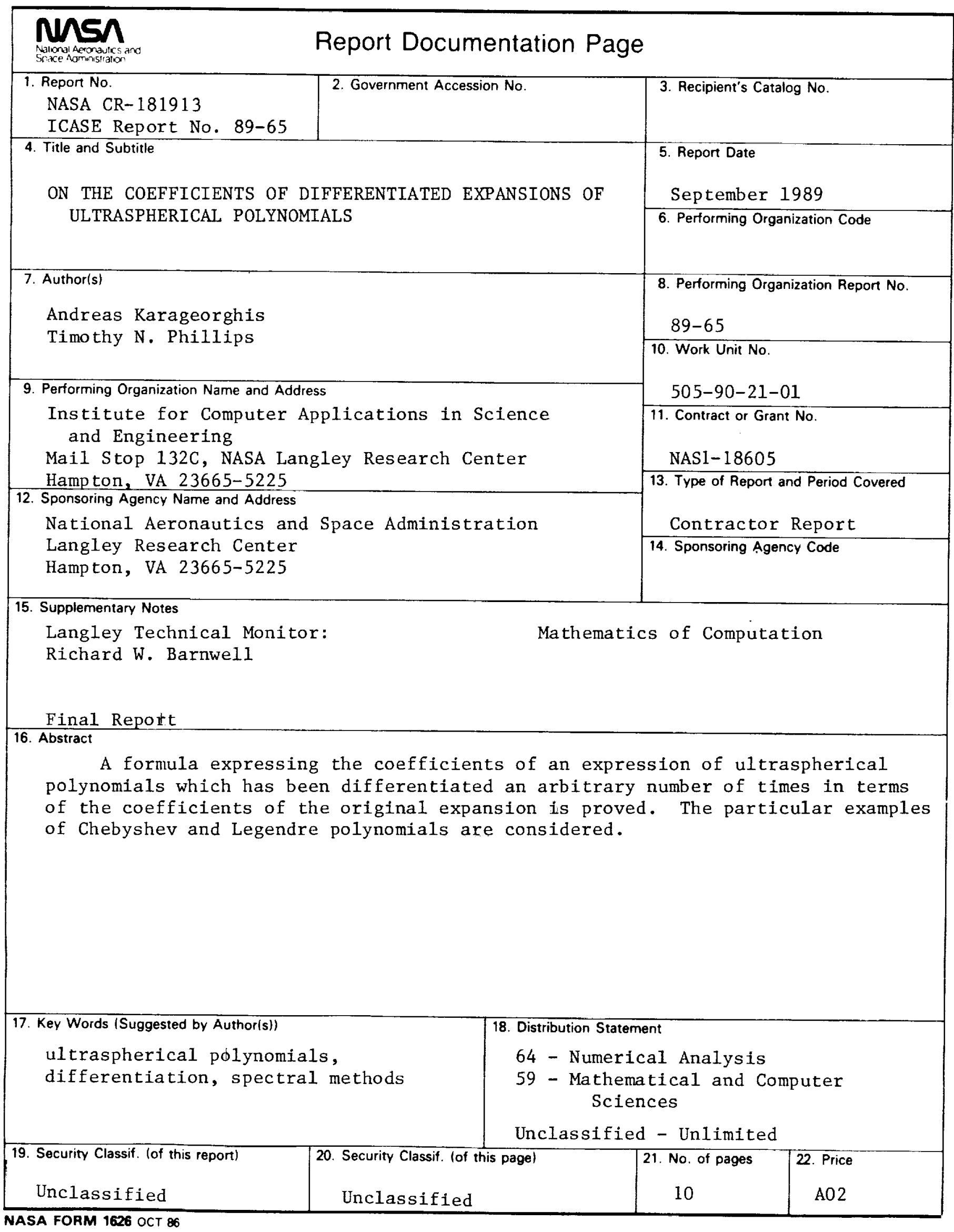


